\title{
SEE cross section calibration and application to quasi-monoenergetic and spallation facilities
}

\author{
Rubén García Alía ${ }^{1, a}$, Markus Brugger ${ }^{1}$, Salvatore Danzeca ${ }^{1}$, Julien Mekki ${ }^{2}$, and Adam Thornton ${ }^{1}$ \\ ${ }^{1} \mathrm{CERN}, \mathrm{CH}-1211$, Genève, Switzerland \\ ${ }^{2}$ Centre National d'Etudes Spatiales (CNES), 18 Avenue Edouard Belin, 31400 Toulouse, France
}

\begin{abstract}
We describe an approach to calibrate SEE-based detectors in monoenergetic fields and apply the resulting semi-empiric responses to more general mixed-field cases in which a broad variety of particle species and energy spectra are involved. The calibration of the response functions is based both on experimental proton and neutron data and considerations derived from Monte Carlo simulations using the FLUKA code. The application environments include the quasi-monoenergetic neutrons at RCNP, the atmospheric-like VESUVIO spallation spectrum and the CHARM high-energy accelerator test facility.
\end{abstract}

\section{Introduction}

In a mixed radiation field (i.e. one composed of different particles species and energies) the number of SEEs $N$ in a given time period will correspond to the convolution of the individual differential particle fluences $\frac{d \phi_{i}}{d E}(E)$ and SEE cross sections $\sigma_{i}(E)$ summed over all the different particle species $i$, as expressed in Eq. 1 .

$$
N=\sum_{i} \int \frac{d \phi_{i}}{d E}(E) \sigma_{i}(E) d E
$$

The cross sections as a function of energy can be expressed as the product of a certain constant cross section value and a weighting function $\omega_{i}(E)$ as shown in Eq. 2, therefore Eq. 1 can be rewritten as Eq. 3 where $\phi_{i}^{e q}$ is defined as the equivalent fluence for the particle species $i$.

$$
\begin{gathered}
\sigma_{i}(E)=\sigma_{i} \cdot \omega_{i}(E) \\
N=\sum_{i} \sigma_{i} \int \frac{d \phi_{i}}{d E}(E) \omega_{i}(E) d E=\sum_{i} \sigma_{i} \phi_{i}^{e q}
\end{gathered}
$$

For the radiation fields that will be treated in this paper, the particle species contributing to SEEs can be divided into two categories according to their interaction mechanisms: thermal neutrons and high energy hadrons (HEH) [1]. Therefore, Eq. 3 can be developed into two terms and resolved for the so-called mixed-field $\mathrm{HEH}$ cross section $\sigma_{H E H}^{*}$ as shown in Eq. 4. For a given mixed-field measurement, this value will depend on the number of measured SEEs, the associated equivalent fluences and the sensitivity to thermal neutrons.

\footnotetext{
ae-mail: ruben.garcia.alia@cern.ch
}

$$
\sigma_{H E H}^{*}=\frac{N-\sigma_{t h} \phi_{t h}^{e q}}{\phi_{H E H}^{e q}}
$$

In the case of the thermal neutrons, SEEs are known to be induced by the neutron capture in ${ }^{10} B$ and the production of ${ }^{7} \mathrm{Li}$ and ${ }^{4} \mathrm{He}$ as ionizing particles. Therefore, the thermal neutron SEE cross section can be expressed as shown in Eq. 5 where $\sigma_{t h}$ is the SEE cross section at 0.025 $\mathrm{eV}$ and $\omega_{t h}(E)$ is defined as shown in Eq. 6.

$$
\begin{gathered}
\sigma_{t h}=\int \omega_{t h} \frac{d \phi_{n}}{d E}(E) d E \\
\omega_{t h}(E)=\left[\frac{0.025 e V}{E(e V)}\right]^{\frac{1}{2}}
\end{gathered}
$$

As to what concerns the HEH contribution and according to experimentally supported nuclear interaction physical considerations that will be developed in the final version of the paper, we assume that for both protons and neutrons, the respective cross sections can be expressed as shown in Eq. 2 where $i$ is either protons or neutrons and $\omega_{i}(E)$ is a three-parameter Weibull function as shown in Eq. 7 where $E_{o}$ in the onset energy and $W$ and $s$ are the shape parameters. In this case the constant cross section term $\sigma_{i}$ corresponds to the saturation value.

$$
w(E)=1-e^{-\left(\left(E-E_{o}\right) / W\right)^{s}}
$$

Therefore, the $\sigma_{H E H}^{*}$ value extracted in a mixed-field through Eq. 4 can be compared to the value retrieved in the monoenergetic measurement set in Eq. 2 which is considered as the calibrated value.

In the work here presented, a set of mixed-field $\mathrm{HEH}$ SEE cross sections are measured and benchmarked against the calibrated value. We will use the ratio $R$ between 
the mixed-field and calibrated HEH cross sections as a means of quantifying how well the given environment is described in terms of SEE induction.

\section{SEE Cross Section Calibration}

\subsection{PSI: protons between 30 and $230 \mathrm{MeV}$}

The ESA Monitor SEU cross section was measured during a set of test campaigns at PSI between September 2011 and April 2014. The Proton Irradiation Facility (PIF) beam line at the Paul Scherrer Institute (PSI) is used extensively by the space community as well as by research groups in other disciplines [2]. Since 2007, the COMET cyclotron is in operation designed to produce a $1000 \mathrm{nA}, 250 \mathrm{MeV}$ proton beam which is currently used in three Gantries and the PIF experimental station. The initial proton energy delivered by the cyclotron during the ESA Monitor calibration tests was $230 \mathrm{MeV}$, which could be degraded through the use of copper plates of different thicknesses.

At PSI, the beam intensity monitoring system consists of an ionization chamber located downstream the degrader. A plastic scintillator placed in the location of the test sample is used to measure the flux before the test runs and establish the conversion factors between the counts from the ionization chamber and the actual proton flux. The same scintillators can be used to extract the beam profile by moving it horizontally and vertically in millimeter steps. The profile is typically homogeneous within $\sim 10 \%$ in a diameter of $5 \mathrm{~cm}$. As to what regards the beam current and respective fluxes, the maximum value is roughly $5 \mathrm{nA}$, corresponding to a flux of $\sim 2 \cdot 10^{8} \mathrm{p} / \mathrm{cm}^{2} / \mathrm{s}$ at 230 $\mathrm{MeV}$ and $\sim 4 \cdot 10^{7} \mathrm{p} / \mathrm{cm}^{2} / \mathrm{s}$ at $30 \mathrm{MeV}$. These values can be reduced linearly with intensity down to a lower limit of roughly $0.1 \mathrm{nA}$.

In order to evaluate the sensitivity spread among individual detectors, eight different monitor were tested at $230 \mathrm{MeV}$, yielding an average cross section value as shown in Eq. 8 and a relative $2 \sigma$ deviation of $7 \%$. This spread was assumed to originate from the sensitivity differences among the detectors, as the count statistics error was significantly smaller in every case (at least $5 \cdot 10^{3}$ SEU counts were accumulated during each run, corresponding to a $2 \sigma$ spread of $\sim 3 \%$ ). Moreover, this spread was assumed to be constant for the different test conditions considered. In addition, a $10 \%$ error in the fluence value provided by the facility is assumed as typically reported.

$$
\sigma_{H E H}=(2.63 \pm 0.32) \cdot 10^{-14} \mathrm{~cm}^{2} / \text { bit }
$$

The ESA Monitor SEU cross section results were normalized to the $230 \mathrm{MeV}$ value and fitted to a threeparameter Weibull function $\omega_{p}(E)$ such as that shown in Eq. 7. The normalized experimental data and the respective response function $\omega_{p}(E)$ are plotted in Fig. 1. In addition to the uncertainly related to the $\sigma_{H E H}$ value (deriving from the sensitivity spread and the fluence measurement) an extra $15 \%$ error is considered for the $\omega_{p}(E)$ function in order to account for the actual energy dependence of the response as opposed to the considered fit.

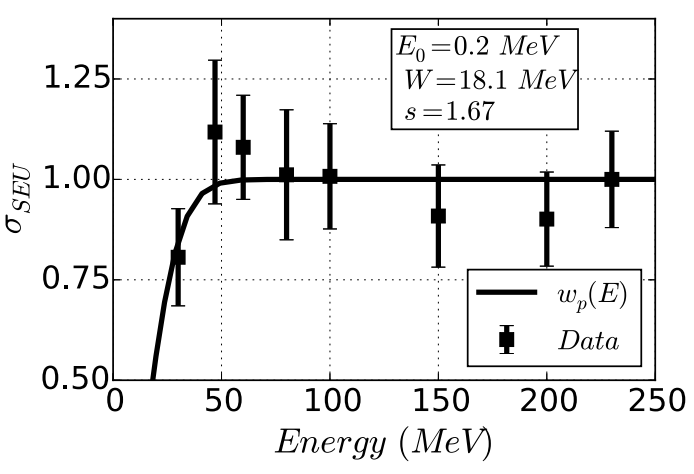

Figure 1. Normalized PSI proton cross section data together with fitted Weibull response function.

\subsection{PTB: Neutrons between 5 and $15 \mathrm{MeV}$}

The PTB Neutron Reference Fields (PIAF) are quasimonoenergetic neutron reference beams in the energy range from thermal to $200 \mathrm{MeV}$ [3]. The main purpose of such beams is the energy response calibration of instruments used for neutron monitoring and dosimetry. The principle behind the production of intermediate and high energy quasi-monoenergetic fields is that of light ions (protons, deuterium) accelerated in a proton or Van-deGraaf (VgG) accelerator impinging on gas or solid low-Z targets (deuterium, tritium, ${ }^{7} \mathrm{Li}$ ). The reactions and field properties of the energies used in the ESA Monitor calibration campaign are shown in Table 1.

Table 1. PTB calibration for different energies in the $1-20 \mathrm{MeV}$ range. Flux rates correspond to a distance of $1 \mathrm{~m}$ from the source. Ti(T) stands for tritiated titanium. The energy spread is represented by the FWHM. The relative contribution of neutrons scattered in the target is indicated by $\Phi_{s c} / \Phi$

\begin{tabular}{c|c|c|c|c|c}
\hline \hline Reaction & $\begin{array}{c}<E_{n}> \\
(\mathrm{MeV})\end{array}$ & $\begin{array}{c}F W H M_{E_{n}} \\
(\mathrm{MeV})\end{array}$ & Target & $\begin{array}{c}\text { Flux } \\
\left(/ \mathrm{cm}^{2} / s\right)\end{array}$ & $\begin{array}{c}\Phi_{s c} / \Phi \\
(\%)\end{array}$ \\
\hline${ }^{2} \mathrm{H}(d, n)^{3} \mathrm{He}$ & 5.0 & 0.2 & $D_{2}$-gas & $5.2 \cdot 10^{3}$ & $<1.0$ \\
${ }^{2} \mathrm{H}(d, n)^{3} \mathrm{He}$ & 8.0 & 0.2 & $D_{2}$-gas & $1.9 \cdot 10^{4}$ & $<1.0$ \\
${ }^{3} \mathrm{H}(d, n)^{4} \mathrm{He}$ & 14.8 & 0.4 & $\mathrm{Ti}(\mathrm{T})$ & $1.3 \cdot 10^{4}$ & 3.0 \\
\hline
\end{tabular}

The measurements of the neutron peak fluence are performed relative to the differential n-p scattering cross section by means of a proton recoil proportional counter (PRPC) or a recoil proton telescope (RCT) depending on the energy. Likewise, the measurement of the spectral fluence is carried out using pulsed beams and the Time-OfFlight (TOF) technique with scintillators and fission ionization chambers. This detection technique cannot be used for low energy neutrons at PTB $(24 \mathrm{keV}-19 \mathrm{MeV})$ due to the beam pulse frequency, therefore their spectral characterization is performed through the unfolding of Bonner sphere readings.

At PTB the ESA SEU Monitor was characterized with the energies reported in Table 1 . These correspond to a range in which the SEE cross section is known to have a strong dependence with energy and that can have an im- 
portant impact on the overall high-energy accelerator SEE rate [1].

The respective normalized SEU cross section values and Weibull response fit $w_{n}(E)$ are shown in Fig. 2 in logarithmic scale and along with the proton response $\omega_{p}(E)$. Similarly to the proton case, a $15 \%$ error is attributed to the use of an analytical fit $\omega_{n}(E)$ as opposed to the actual response. These results are regarded as monoenergetic for SEU calibration purposes as (i) the proportion of scattered neutrons is at the percent level (ii) the cross section decreases rapidly with energy in this range.

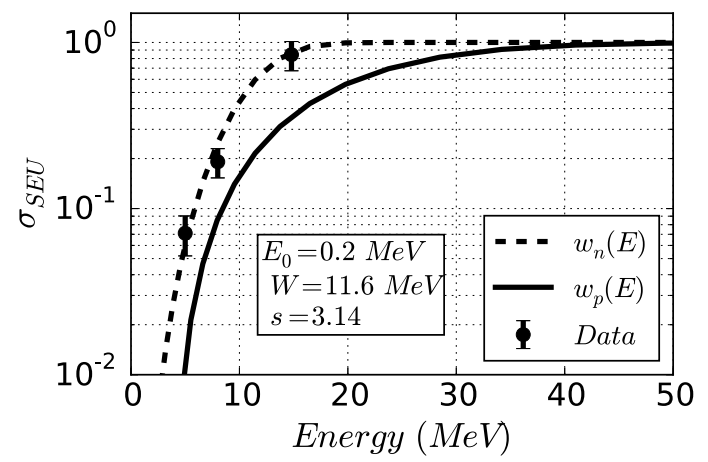

Figure 2. Normalized PTB neutron cross section data together with the fitted Weibull response function.

\section{Application to quasi-monoenergetic and spallation facilities}

\subsection{RCNP: quasi-monoenergetic neutrons at 100 and $300 \mathrm{MeV}$}

At RCNP, quasi-monoenergetic neutron beams in the 80$400 \mathrm{MeV}$ range are provided through the ${ }^{7} \mathrm{Li}(p, x n)$ reaction [4]. A $1 \mathrm{~cm}$ thick enriched Li target is used to produce the neutrons. An NE213 liquid scintillator was used to detect the generated neutron spectra using a TOF technique. The proton energy during the ESA Monitor calibration experiments performed in November 2014 was determined to be 100 and $296 \mathrm{MeV}$, yielding neutron peak energies of 96 and $293 \mathrm{MeV}$ respectively. As a figure-of-merit of the contribution of scattered neutrons to the total spectrum, the ratio between the peak and total above $3 \mathrm{MeV}$ fluences is used, $\Phi_{\text {peak }} / \Phi_{>3 \mathrm{MeV}}$, which was reported to be equal to 0.41 for the $100 \mathrm{MeV}$ case and 0.44 for the $296 \mathrm{MeV}$ case. The beam intensity is monitored using a Faraday cup collecting the protons after interacting with the production target.

The measured spectra as reported by the facility are shown in Fig. 3. The response function $w_{n}(E)$ is convoluted with the neutron fluxes $\phi_{n}(E)$ in order to yield the $\phi_{H E H}^{e q}$ from Eq. 3 in which the thermal neutron contribution is assumed to be negligible. The resulting cross sections are shown in Table 2 for the quasi-monoenergetic RCNP neutron beams with their associated uncertainty. The latter considers the count statistics, the sensitivity spread, a $10 \%$ margin in the neutron flux measurement and a $15 \%$ uncertainty related to the use of $w_{n}(E)$ as a response function. The normalized cross section results are plotted in Fig. 4. As can be seen, the 96 and $293 \mathrm{MeV}$ neutron values are $27 \%$ and $5 \%$ larger than the saturation value, respectively; and therefore within the considered statistical uncertainty. This is compatible with the fact that as will be shown in the final version of the paper through Monte Carlo simulations, in virtue of their similar nuclear reaction cross section protons and neutrons are expected to yield equivalent SEE cross sections above roughly 50 $\mathrm{MeV}$.

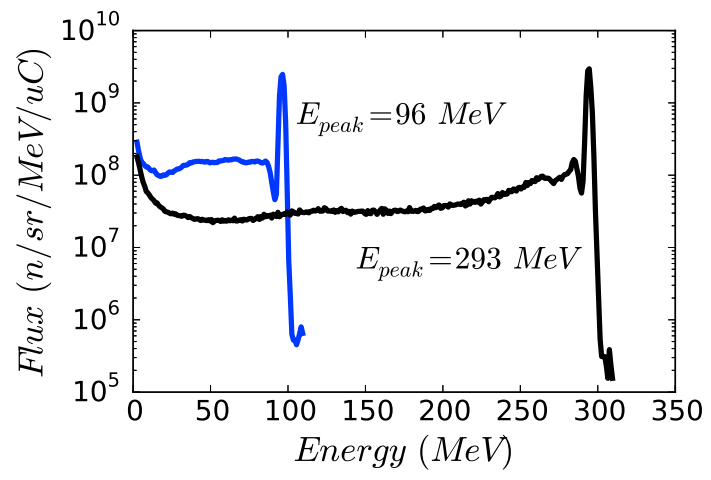

Figure 3. RCNP spectra for 100 and $296 \mathrm{MeV}$ as measured through the NE213 TOF technique.

Table 2. SEU cross section summary for the ESA SEU Monitor at RCNP.

\begin{tabular}{c|c|c}
\hline $\begin{array}{c}\text { Energy } \\
(\mathrm{MeV})\end{array}$ & $\begin{array}{c}\sigma_{S E U} \\
\left(\cdot 10^{-14} \mathrm{~cm}^{2} / \text { bit }\right)\end{array}$ & $\begin{array}{c} \pm 2 \sigma \\
(\%)\end{array}$ \\
\hline \hline 96 & 3.36 & \pm 26 \\
293 & 2.77 & \pm 26 \\
\hline
\end{tabular}

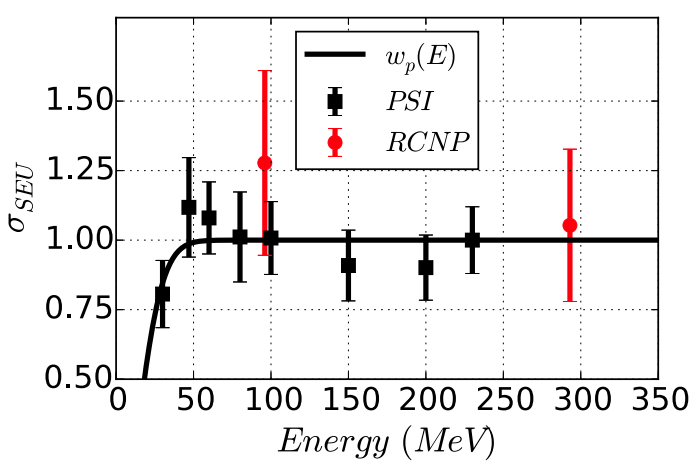

Figure 4. Normalized RNCP neutron quasi-monoenergetic cross section data together with the fitted Weibull proton response function. 


\subsection{VESUVIO: Atmospheric-like neutron spectrum}

The VESUVIO neutron beam is part of the ISIS-STFC laboratory in Oxford, UK. Despite its main use as a condensed matter research instrument [5]. Benchmark measurements have been performed proving the VESUVIO provides a neutron spectrum similar to the ambient at sea level. Neutrons are generated through the interaction of a $800 \mathrm{MeV}, 2 \mu \mathrm{A}$ proton beam with a spallation target. The proton beam is accelerated in a synchrotron as two 100 ns long pulses with a frequency of $50 \mathrm{~Hz}$. The VESUVIO beamline is at $60^{\circ}$ with respect to the initial proton beamline. The neutron flux obtained above $10 \mathrm{MeV}$ is $\sim 5.8 \cdot 10^{4} / \mathrm{cm}^{2} / \mathrm{s}$ therefore roughly an order of magnitude lower than those available at TRIUMF or LANSCE. The neutron spectrum is calculated using the MCNPX Monte Carlo simulation tool and benchmarked against TOF measurements performed with different detectors including Bonner spheres, activation foils, CCD devices and Thin Film Breakdown Counters (TFBC). The flux measurement during the VESUVIO experiments relies on the benchmarked MCNPX calculations scaled with the beam current.

Concerning the ESA SEU Monitor measurements performed in March 2014, two configurations were used: one with the primary neutron VESUVIO beam and one with a $1.5 \mathrm{~mm} \mathrm{Cd}$ foil surrounding the detector in order to absorb the thermal neutrons by means of the roughly $7000 \mathrm{~b}$ associated capture cross section. The two concerned spectra can be seen in Fig. 5 as reported by the facility through benchmarked MCNPX simulations.

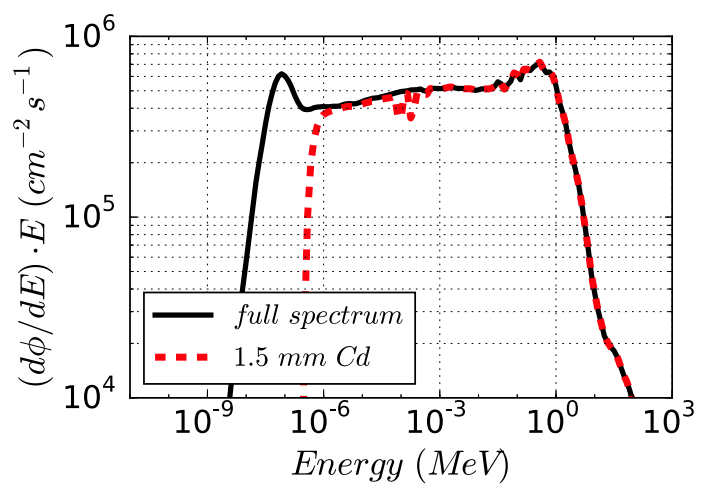

Figure 5. VESUVIO lethargy flux spectrum with and without the Cadmium absorber.

As the ESA Monitor is known to be sensitive to thermal neutrons and provided the high energy neutron spectrum remains unaltered when introducing the cadmium absorber, the thermal neutron SEU cross section $\sigma_{t h}$ can be extracted through the ratio of the differences of the SEU rates (SER) and the respective equivalent thermal neutron fluxes $\phi_{t h}^{e q}$, without and with absorber, as shown in Eq. 9.

$$
\sigma_{t h}=\frac{S E R_{f u l l}-S E R_{C d}}{\phi_{t h}^{e q(f u l l)}-\phi_{t h}^{e q(C d)}}=(2.30 \pm 0.62) \cdot 10^{-15} \mathrm{~cm}^{2} / \mathrm{bit}
$$

The HEH cross section can therefore be derived from Eq. 3 yielding the result shown in Eq. 10.

$$
\sigma_{H E H}^{*}=(2.79 \pm 0.60) \cdot 10^{-14} \mathrm{~cm}^{2} / \text { bit }
$$

When divided by $\sigma_{H E H}$ the resulting ratio is $1.06 \pm$ 0.27 therefore the VESUVIO cross section value is fully compatible with the calibration.

\subsection{CHARM: high-energy accelerator like mixed field}

In the final version of the paper, results for the CHARM accelerator-like mixed field facility at CERN will be shown for different particle spectra, concentrating on the impact of the hadron species (mainly protons, neutrons and pions) as well as their energy distribution.

\section{Conclusions and Outlook}

Results for the monoenergetic calibration of the ESA SEU Monitor are shown and applied to various mixed-field cases. For all three conditions (100 and $300 \mathrm{MeV}$ quasimonoenergetic neutron fields at RCNP and spallation neutron spectrum at VESUVIO) the figure-of-merit $R$ defined as the ratio between the mixed-field and reference $\mathrm{HEH}$ cross sections is compatible with 1 . Therefore, this method provides both a general approach and specific calibration results that can be used to determine the degree of accuracy in which a mixed radiation fields is described in terms of capability of inducing SEEs.

\section{References}

[1] K. Roed et al., IEEE Trans. Nucl. Sci. 59, 1040 (2012)

[2] W. Hajdas et al., Radiation Effects Data Workshop 160 (2002)

[3] S. Röttger et al., 4th International Workshop on $\mathrm{Nu}$ clear Fission and Fission-Product Spectroscopy 1175 375 (2009)

[4] Y. Iwamoto et al., Nuclear Instruments and Methods in Physics Research Section A: Accelerators, Spectrometers, Detectors and Associated Equipment 804, 50 (2015)

[5] C. Andreani et al., Applied Physics Letters 92, 114101 (2008) 